\title{
Laboratory Vendor Name
}

National Cancer Institute

\section{Source}

National Cancer Institute. Laboratory Vendor Name. NCI Thesaurus. Code C82537.

The literal identifier of the laboratory that performs an assessment or examination. 